Aus der Medizinischen Universitäts-Poliklinik in Leipzig (Direktor: Geh. Med.-Rat Prof. Dr. Hoffmann), Abteilung für Hautkranke (Assistent: Dr. Hans Vörner).

\title{
Ueber eine neue Anwendungsweise der konzentrierten Karbolsäure in der ex- ternen Therapie, vor allem bei Bubonen und Furunkulose.
}

Von cand. med. Werner Wolff.

Die konzentrierte Karbolsäure wird in der Hautstation der hiesigen Medizinischen Universitäts-Poliklinik, an der ich meine Beobachtungen gemacht habe, häufig benutzt, und wir haben zum Teil Verfahren angewandt, die schon von früher her bekannt sind, zum Teil eigene Methoden ausgebaut. Am bekanntesten dürfte wohl die Behandlung des Ulcus molle mit reiner Karbolsäure nach Herff-Neisser (1) sein, und auch wir haben hier eine grofe Anzahl mit gutem Erfolge behandelt, Fälle, auf die ich wegen der Bekanntheit des Verfahrens jedoch nicht näher eingehen will. Neu dürfte die von uns viel angewandte Karbolsäurebehandlung der durch Ulcus molle entstandenen Bubonen sein. Zwar ist schon früher einmal von Harvey und Taylor (2) Karbolsäure zu ihrer Behandlung empfohlen worden, jedoch nur in verdünnter, $6 \%$ iger Lösung ( 3 Gran Karbolsäure auf 1 Unze $\mathrm{H}_{2} \mathrm{O}$ ), von der 20-30 Minims eingespritzt werden. Außerdem muf nach der Injektion der Bubo durch einen Sand- oder Schrotbeutel beschwert werden.

Was die von uns hier in der Poliklinik und von Herrn Dr. Vörner in seiner Privatpraxis schon seit längerem geübte Methode anlangt, so will ich im folgenden etwas näher auf diese eingehen, da sie noch unbekannt, aber in der Sprechstunde des praktischen Arztes leicht anzuwenden ist, außerdem den besonderen Vorteil hat, daß die Patienten nötigenfalls auf diese Weise auch ambulant behandelt werden können.

Bei der Behandlung kommt hauptsächlich in Betracht, ob der Bubo noch geschlossen und hart, fluktuierend oder bereits durchgebrochen ist. Die geschlossenen werden zunächst am ersten Tage im Bereich der Schwellung mit einem Anstrich von reiner Karbolsäure versehen, die mittels eines mit Watte umwickelten Stäbchens in einem etwa $0,5-1 \mathrm{~cm}$ breiten Strich 
einmal aufgetragen wird.1) Am andern Tage kann das Verfahren wiederholt und in den nächsten Tagen fortgesetzt werden, bis die Haut anfängt sich abzuschuppen. Dann macht man einige Tage Pause und beginnt die Anstriche von neuem, falls der Bubo noch nicht zurückgegangen ist. Der fluktuierende Bubo wird ebenfalls zwei bis drei Tage bestrichen, am vierten, wenn die Fluktuation nicht geschwunden, eine Inzision von etwa $2 \mathrm{~cm}$ Länge gemacht, der Eiter abgelassen, die Absceßhöhle mit reiner Karbolsäure ausgestrichen, dann verbunden und jeden zweiten bis dritten Tag das Verfahren wiederholt, bis die Höhle granuliert, dann mit indifferentem Verband (Salbe oder Jodoformgaze) bis zur Abheilung behandelt. Ist der Bubo bereits durchgebrochen, so ist die Behandlung die gleiche wie beim eröffneten.

Von den in den Jahren 1904 und 1905 so behandelten Fällen seien nur einige herausgegriffen.

Fall 1. Karl N., vor 14 Tagen typisches Ulcus molle. Kommt jetzt mit einem etwa $6 \mathrm{~cm}$ langen, $3 \mathrm{~cm}$ breiten, nicht fluktuierenden, harten Bubo, der nach zweimaliger Bestreichung, ohne Oeffnung, zurückgeht.

Fall 2. Heinrich B., Kontorist. Seit einigen Tagen Ulcus am Bändchen, an das sich nach einigen Tagen rechtseitiger Bubo anschließt, der nach Bestreichung am 22., 25. und 30. September 1905 ohne Eröffnung zurückgegangen ist.

Fall 3. Hermann B. (Journal-No. 542/05). Kommt mit verschiedenen Ulcera mollia am Präputium und einem linkseitigen Bubo. Letzterer wird zunächst mit Karbolsäure betupft in Gestalt eines $0,5 \mathrm{~cm}$ breiten Striches. Am dritten Tage wiederholt, daraufhin Rückgang. Nach Rückgang des Bubo bleibt Patient weg, die Ulcera heilen aber wegen der gleichzeitig bestehenden Phimose schlecht ab, sodaß Patient nach einem Monat mit einem neuen, diesmal rechtseitigen Bubo wiederkommt, der dreimal in Abständen von drei bis vier Tagen betupft wird und daraufhin zurückgeht. Vier Monate später ist links wiederum ein neuer Bubo entstanden, der nach viermaliger Bestreichung zurïckgeht. Die Ulcera waren nur teilweise zu behandeln, da ein Teil unter der phimotisch verengten Vorhaut einer Behandlung nicht zugänglich war.

In gleicher Weise wie die Bubonen mittels Anfstreichung reiner Karbolsäure haben wir auch einen Fall von Periadenitis axillaris behandelt.

August W., Maurer, kommt mit einem über walnußgroßen, lappigen, derben, sehr schmerzhaften Tumor in der Achselhöhle, der am 8., 9. und 13. August 1904 mit Karbolsäure bestrichen wurde, am 18. August schon zu Erbsengröße zurückgegangen war, sodaß Patient nach nochmaliger Tuschierung am 20. August als geheilt entlassen werden konnte.

Besonders gute Erfolge haben wir mit der reinen Karbolsäure bei einer der Periadenitis ähnlichen Hautaffektion erzielt: der Furunkulose, wo wir ein Verfahren angewandt haben, das von meinem Chef, Herrn Dr. Hans Vörner, zuerst angegeben und von ihm bereits seit Jahren in seiner Privatpraxis viel angewandt ist, und das sich vor den bisher bekannten durch seine große Einfachheit auszeichnet.

Auf den Wert der konzentrierten Karbolsäure bei dieser Krankheit hat schon Lister (3) 1867 aufmerksam gemacht, indem er mit Karbolöl (1:4) getränkte Läppchen auf die Knoten legte, während Eade (4) erst den noch nicht eröffneten Karbunkel inzidiert und dann die Inzisionswunde mit in Karbolöl getränkten Leinenfäden tamponiert, ein Verfahren, das Eames (5) 1873 modifizierte, der, um ein möglichst allseitiges Eindringen der Flüssigkeit zu erreichen, das warme Karbolöl nach tiefer Inzision einspritzte. In späterer Zeit, 1887, empfahl Bidder (6), nach 25jähriger Erprobung, zu gleichem Zwecke die 2\% ige Karbolsäurelösung, von der er, ohne vorher zu inzidieren, einmalig eine Pravaz-Spritze mittels zwei bis vier Einstichen in den Eiterherd injizierte, eine Methode, die nach ihm dann auch noch Leu (7), Clever (8) und Arnaudet (9) empfahlen, während Murray (10) vorschlug, reine Karbolsäure tief in den Karbunkel zu injizieren.

Von all diesen Methoden unterscheidet sich die an der hiesigen Klinik geübte Vörnersche Methode wesentlich, da bei ihr die schmerzhaften Injektionen durchaus unnötig sind. Wir unterscheiden zunächst zwischen unerweichten und

1) Zur Betupfung benutzen wir eine konzentrierte Lösung von Acid. carbolic. crystallisat. (9 Teile) in absolutem oder rektifiziertem Alkohol (1 Teil), da diese Lösung der kristallisierten Säure in Alkohol einmal wirksamer ist als Acid. carbolic. per aquam liquefactum, dann aber auch den Vorzug besitzt, nicht wie dieses in erheblicher Weise zu dunkeln. mehr oder weniger fluktuierenden Knoten und zwischen kleineren und größeren. Zur Behandlung der unerweichten kleinen Furunkel genügt eine zentrale Betupfung mit Acid. carbolic. per aquam oder, aus den schon vorher genannten Gründen, besser per alcoholem liquefactum. Bei größeren hat es sich als zweckmäßig erwiesen, mit einer feinen Nadel oder Sonde die zentral gelegenen Haarbälge. bzw. Talgdrüsenöffnungen so tief als möglich zu tuschieren. Ist, wie häufig, ein zentraler Kanal auffindbar, so wird in denselben so tief als möglich das Medikament mit einem der Dicke des Kanals entsprechenden Instrumente hineingebracht. Bei erweichten Knoten benutzt man entweder die natürliche Oeffnung oder legt künstlich, durch einen Einstich, eine solche an, die nicht größer zu sein braucht, als zum Abfluß des Eiters unbedingt notwendig ist, und durch die dann die Tuschierung der Höhle - einmal täglich - erfolgt. Meist genügt eine einzige Betupfung, manchmal hat man das Verfahren mehrere Tage hintereinander zu wiederholen, doch haben wir auch bei unseren größten Furunkeln nicht mehr als zehn Betupfungen gebraucht. Im allgemeinen kann man sagen, je größer der Furunkel, desto länger die Behandlung; doch ist es im Interesse einer rascheren Heilung dabei vorteilhafter, die Behandlung dann täglich vorzunehmen, anstatt intermittierend an jedem zweiten oder dritten Tage.

Im übrigen besteht die Behandlung darin, dab am ersten und zweiten Tage, namentlich bei erheblicher Schwellung, Umschläge verordnet werden, wozu wir meist die Vörnersche (11) Lösung verwandt haben (Acidi borici 4,0, Liqu. alum. acet. ad 100,0, die in dieser Zusammsetzung lange Zeit hindurch ohne Niederschlag haltbar ist), worauf eine indifferente Salbe, wie Bor- oder Arg. nitric.-Salbe aufgetragen wird. Pflaster sind nicht so empfehlenswert, wegen der unter diesen eventuell eintretenden Schwellung, jedoch kann man vielfach auf jeden Verband verzichten. Die ganze Technik, welche zu dieser Behandlung gehört, verursacht keine Schwierigkeiten. Einzig und allein hat man noch darauf $\mathrm{zu}$ achten, das watteumwickelte Stäbchen nicht $\mathrm{zu}$ tief einzutauchen, um beim Tuschieren ein Ueberfließen der Karbolsäure auf das gesunde Gewebe zu vermeiden.

An der hiesigen Poliklinik haben wir während der letzten zwei Jahre ungefähr 50 Fälle, teils einfacher Furunkel, teils multipler Furunkulose, zu Behandlung bekommen.

Von den Furunkeln beanspruchten 12 eine eintägige Behandlung, bei 5 Fällen genügte ein $\mathrm{zw}$ e imaliges Betupfen, und nur in vier Fällen mußten wir die Behandlung auch ein drittes Mal wiederholen. Es handelte sich in der Hauptsache, in acht Fällen, um kleine Furunkel im Nacken von Erbsen- bis Kirsch- und Bohnengröße, von denen nur zwei erst inzidiert werden inußten, während bei je drei ein ein - und zweimaliges bloßes Betupfen genügte.

Die einmalige Betupfung genügte ferner bei kleineren, unerweichten Furunkeln am Arm (zwei Fälle, davon einer von Kirschgröße), am Unterkiefer (ein Fall), bei einem kirschgroßen der linken Backe, bei einem bohnengroßen am Knie und bei je einem kleineren am Ober- und Unterschenkel, eine zweimalige bei drei Nackenfurunkeln, und in einem Falle, wo Patient mit einem, im Zentrum vereiterten Furunkel der Zungenspitze und starker Schwellung der Zunge in Behandlung kam. Kleine Inzision zur Entleerung des Eiters, Auswischung mit Karbolalkohol, die am folgenden Tage wiederholt wurde, wobei schon nach der erstmaligen Behandlung ein deutlicher Rückgang der Schwellung zu bemerken war, sodaß Patient nach zwei weiteren Tagen, an denen jedoch nicht mehr geätzt wurde, als geheilt aus der Behandlung entlassen werden konnte. - In einem Falle von Lippenfurunkel, wo Patient mit einer seit acht Tagen bestehenden, im Zentrum fluktuierenden, schmerzhaften Schwellung der rechten Oberlippe $\mathrm{kam}$, war nach der erstmaligen, zentralen $\mathrm{Be}-$ tupfung am folgenden Tage Spontanaufbruch erfolgt, sodaß man durch die Oeffnung in eine etwa kirschgroße Eiterhöhle gelangte, die wiederum mit Karbolsäure ausgewischt wurde, sodaß der Karbunkel nach drei Tagen abheilte.

Von vier Fällen, wo wir eine dreimalige Betupfung anwenden mußten, handelte es sich bei zwei Patienten um je einen kirschgroßen Furunkel am Unterarm, bei einem um einen bohnengroßen im Nacken und bei dem vierten Kranken um einen unvereiterten, walnußgroßen Furunkel am Gesäß. Alle heilten nach dreimaliger, einen Tag um den andern erfolgten Betupfung glatt ab.

Während in den bisher erwähnten Fällen diese kurzdauernde Einwirkung zur Beseitigung der Furunkel genügte, nahm bei drei 
Patienten die Behandlung etwas längere Zeit in Anspruch: In einem Falle handelte es sich dabei um je einen walnußgroßen Furunkel am Bauch und am Arm, von denen letzterer stark erweicht, ersterer dagegen noch hart war und die beide durch eine sechs Tage hintereinander erfolgte Betupfung ohne Eröffnung zum Schwinden gebracht wurden, beim zweiten Patienten um einen etwa $5 \mathrm{~cm}$ im Durchmesser betragenden Karbunkel im Nacken, der nach viertägiger Tuschierbehandlung zurückging, und im letzten Falle um einen erweichten Furunkel von Walnußgröße am Rücken, bei dem nach geringer Inzision, Entleerung des Eiters und täglicher Auswischung der Höhle mit Karbolsäure vom 7.-10. Januar 1905, am 14. ein wesentlicher Rückgang und am 19. Heilung konstatiert werden konnte.

Von 14 Fällen von multipler Furunkulose bei Erwachsenen genügte in fünf Fällen ebenfalls eine einmalige Betupfung. Es handelte sich dabei in der Hauptsache um eine Anzahl kleinerer, meist erbsengroßer Furunkel auf dem Unterarme, verbunden mit pustulösem Ekzem, auf der Vorder- und Rückseite des Stammes, am After, auf dem behaarten Kopf und im Gesicht, wobei ein gleichzeitig vorhandener, besonders großer an der rechten Schläfe, dessen Basis $3 \mathrm{~cm}$ im Durchmesser hatte, ebenfalls durch einmalige Betupfung zum Schwinden gebracht wurde. In fünf Fällen mußte die Behandlung auf drei bis fünf Tage ausgedehnt werden (je ein Fall von Furunkulose am Stamm, Rücken, Glutaeen, und zwei am Unterarm), bei zwei Fällen von Furunkulose des Vorderarms auf sechs bis sieben Tage (wobei der eine Patient bereits seit einem Vierteljahre daran litt, und der Vorderarm dicht von zahlreichen kleinen Furunkeln besetzt war, während bei dem zweiten Patienten sich neben den kleineren mehrere kirschgroße Furunkel vorfanden) Nur in zwei Fällen seit Jahren bestehender Furunkulose (einer an den Glutaeen, der andere an den Beinen, dabei beide ziemlich walnu(3groß) beanspruchte die Behandlung zehnmalige, tägliche Aetzung bis zum völligen Schwunde.

In gleicher Weise haben wir eine Anzahl - fünf - von Säug lingen mit multipler Furunkulose des Kopfes und ganzen Körpers behandelt, von denen in zwei Fällen die Eruptionen nach einmaliger, in zwei anderen Fällen nach drei- bis siebenmaliger, in Zwischen räumen von je zwei Tagen ausgeführter Betupfung schwanden. In einem besonders schweren Falle, einem elf Wochen alten Kinde, dessen ganzer Körper von zahllosen Furunkeln jeden Alters und jeder Größe dicht besetzt war, wurden am ersten Tage nur die am schlimmsten vereiterten Furunkel und diejenigen, die am frischesten waren, betupft, etwa ein Drittel der Gesamtzahl. Täglich fortbehandelt, stets etwa ein Drittel der vorhandenen, sodaß nach vier Wochen die Affektion so gut wie beseitigt war. Die folgenden vier Wochen wurde das Kind nur zweimal wöchentlich besichtigt, und es fand sich nur gelegentlich ein neu sich entwickelnder Furunkel, der dann sofort beseitigt wurde, soda 3 sich nach acht Wochen seit Beginn der Behandlung keine Eruption mehr zeigte, und das Kind nach etwa 40 Konsultationen als geheilt aus der Behandlung entlassen werden konnte.

Auch bei drei Fällen von rezidivierender Furunkulose haben wir die gleiche Behandlungsweise angewendet, und in allen drei Fällen mit dem Erfolge, daß die Patienten, die früher ständig daran litten, seit über einem halben Jahre von Furunkeln völlig verschont blieben.

Die guten Erfolge bei Bubonen und Furunkulose veranlaßten uns, das Verfahren auch bei anderen, ähnlichen Hautaffektionen zu versuchen. So haben wir in gleicher Weise durch einfache Betupfung mit in Alkohol gelöster, konzentrierter Karbolsäure die infektiösen Krankheiten der Mundhöhle, Stomatitis aphthosa und ulcerosa (Stomakace) behandelt, und hierbei die gleichgünstigen Resultate erzielt. Auch hier wieder einige Krankengeschichten als Belege.

Fall 1. Otto R. (No. 606/05). Stomatitis simplex ulcerosa. Seit etwa fünf Wochen leidet Patient an einer schweren Entzïnddung des Zahnfleisches, bei der die bisher angewandten Mittel (Desinfizientien und Adstringentien) ohne Erfolg gewesen sind. Die Zähne, an denen viel Zahnstein, sind locker, das Zahnfleisch schwärzlich verfärbt, wulstig, zum Teil der Schleimhaut beraubt, zum Teil abgelöst und mit eitrigen Massen belegt. In gleicher Weise ist die Wangenschleimhaut verändert. Zweimalige Betupfung am ersten und dritten Tage bringt alle Symptome innerhalb acht bis zehn Tagen zurück.

Fall 2. Wilhelm N, 40 Jahre alt (No. 552/05). Stomakace. Patient ist seit ungefähr einem Monat an einer schweren Entzündung des Zahnfleisches erkrankt. Es zeigte sich bei der Untersuchung, daß das Zahnfleisch an den Rändern aufgelockert ist, sodaß die Wurzeln der Zähne stärker. sichtbar sind. Dabei macht dasselbe den Eindruck von Granulationen, zwischen denen hier und da kleine Geschwüre vorhanden sind." Zwischen Zahnfleisch und
Zähnen dringt ein übelriechendes Sekret hervor. Die geschwollenen Partien sind weich und blaurot verfärbt. Die kranken Partien werden mit Karbolsäure betupft, die Behandlung am zweiten, vierten und siebenten Tage wiederholt, nebenbei Spülung mit Boraluminium. Nach 14 Tagen geheilt.

Fall 3. Hermann Pf. (No. 256/04). Ulcus aphthosum mucos. labii superioris. Kein Anhaltpunkt für Lues. Zeigt gegenüber einem defekten Molarzahn auf der Schleimhaut der Oberlippe eine mit dickem Eiter belegte, pfennigstiickgroße Stelle mit stark entzündlichem Rande. Zweimalige Karbolsäurebetupfung führt zur Heilung.

Von weiteren. Krankheiten, die wir an der Poliklinik mit konzentrierter Karbolsäure behandelt haben, sei auch das Scrofuloderma genannt. Bekanntlich heilen die Scrofulodermen auch spontan, bedürfen hierzu aber einiger Zeit und führen gewöhnlich zu ausgedehnter Narbenbildung. Die Behandlung beschleunigt die Heilung und verhindert eine größere Entstellung. Wenn auch die chirurgische Behandlung entschieden noch die rationellste ist, so gibt es doch messerscheue Patienten, die von einer Operation nichts wissen und anderseits ihre Tätigkeit wegen einer solchen nicht unterbrechen wollen. Für diese kommt die von uns geübte Karbolsäurebehandlung vor allem in Betracht. Die noch unerweichten Scrofulodermen werden gleich den Bubonen mehrmals tuschiert, die erweichten werden inzidiert, das abgestorbene Gewebe eventuell mit scharfem Löffel entfernt, und nun die Herde, wenn möglich, bis zu acht Tagen hintereinander, täglich einmal mit der konzentrierten Karbolsäure gleich den eröffneten Bubonen und Karbunkeln - tuschiert, hierauf ein indifferenter Salbenverband angelegt, unter dem der Herd je nach seiner Größe in kürzerer oder längerer Frist glatt abheilt. Die gleiche Methode kommt auch für skrofulöse und tuberculöse Drüsen in Betracht, jedoch sind Fälle dieser Art in der Poliklinik in letzter Zeit nicht behandelt worden; aber ich verweise auf die Arbeit, die Herr Dr. Vörner selbst über derartige Fälle veröffentlicht hat (12). Hier sei nur über zwei noch nicht referierte Fälle der vorher erwähnten Art berichtet, die Herr Dr. Vörner mir in liebenswürdiger Weise zur Veröffentlichung überlassen hat.

F'all 1. Gertrud T., Dienstmädchen, kommt am 2. September 1905 mit einem markstückgroßen Scrofuloderma am Halse mit Erweichung der rechten Submaxillardrüse. Nachdem sie zwölfmal mit Karbolsäure behandelt worden war, konnte sie am 1. Oktober aus der Behandlung entlassen werden; am 12. Oktober stellte sie sich wieder vor, wo Abheilung erfolgt war.

Fall 2. Richard R., kommt mit einem ovalen, etwa $4 \mathrm{~cm}$ langen und $2 \mathrm{~cm}$ breiten Scrofuloderma am horizontalen Unterkieferast, das leicht ausgeschabt und am 24., 25., 26. und 28. September 1905 mit Karbolsäure betupft wurde, wonach glatte Abheilung erfolgte.

Die gleiche Behandlungsweise haben wir auch bei zwei Fällen von Panaritium und einem Fall von Kerion celsi angewandt.

Es handelte sich dabei um je ein Panaritium am Mittel- und Zeigefinger, die beide nach dreimaliger Betupfung mit Karbolsäure, unter gleichzeitigen Umschlägen mit Boraluminium, glatt abheilten; der dritte Patient mit Kerion celsi hatte am Unterkiefer eine Geschwulst, deren Basis 4:7 cm, deren Höhe $1,5 \mathrm{~cm}$, von derber Konsistenz, starker Empfindlichkeit und intensiv roter Farbe, die auf Druck verschwindet. Herausgezogene Haare weisen Trichophyton tonsurans auf. Zweimalige Betupfung mit Karbolsäure, im übrigen Umschläge mit Boraluminium, worauf Abheilung in acht Tagen erfolgte.

Wir haben in letzter Zeit erst ein zehnjähriges Kind mit einem händtellergroßen, feuerroten, nicht erbabenen Naevus, der von der Gegend hinter dem Ohr bis zur Clavicula reicht, durch wöchentlich einmalige Betupfung während eines halben Jahres zur Abheilung gebracht, und augenblicklich verwende ich an mir selbst die Karbolsäure zur Beseitigung einer Sykosis der Oberlippe, die sich äußert in starker Schuppung der Epidermis und Bildung von kleinen Eiterpusteln an der Austrittsstelle der Schnurrbarthaare, indem ich die kleinen Eiterpusteln eröffne, wenn möglich, durch Epilation des betreffenden Haares, den Eiter herausdrücke und nun mittels eines in Karbolsäure getauchten Holzzahnstochers die be. treffende kleine Stelle verätze, sodaß ich durch die seit 14 Tagen so geäbte Behandlung schon eine bedeutende Besserung, Verminderung der Desquamation und Pustelbildung, erzielt habe. 
1824

DEUTSCHE MEDIZINISCHE WOCHENSCHRIFT.

No. 45

Wenn auch unsere Versuche über die weitere Anwendung der konzentrierten Karbolsäure noch nicht abgeschlossen sind, so würde es mich doch freuen, wenn schon diese Zeilen ihren Zweck erfüllten, gerade den praktischen Arzt für unsere Methode $\mathrm{zu}$ interessieren, von deren Zuverlässigkeit er sich durch eigene Anwendung bald selbst überzeugen dürfte.

Literatur: 1. v. Herff, Behandlung des weichen Schankers bei Frauen mit konzentrierter Karbolsäure. Monatshefte für Geburtshilfe und Gynăkologie 1895, S. 577. A. Neisser, Bemerkungen zur Therapie des Ulcus molle. Berliner klinische Wochenschrift 1895 , Bd. 32 , No. 36 . - 2. Philipp Harvey, The abortive treatment of bubo by the methode of Morse K. Taylor. Americ. med. news 1886, S. 98. 3. Lister, Ueber eine neue Behandlungsmethode von komplizierten Frakturen und Abscessen. Lancet $1867,11-17$, II. 4. - 4. Peter Eade, Ueber die orthche BehandKarbolinjektionen gegen tiefsitzende Entzündungen. British medical Journal, Mai 1873 , S. 490 . - 6. A. B id der (Berlin), Ueber Abortivbehandlung des Furunkels mittels parenchymatőser Injektion. Berliner klinische Wochenschrift 1887, No. 14. Die abortive Behandlung des Furunkels (Karbunkels) mit Hilfe subcutaner Desinfektion. Deutsche medizinische Wochenschrift 1902, No. 18 11. 19. - 7. Leu, Ein Beitrag zur

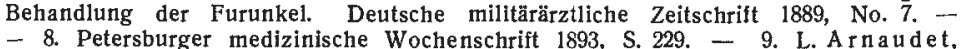
Note sur le treatment du furoncle et de l'anthrase. L'Union médic. 1888, No. 15, S. 177 . - 10. Alan B. Murray, The treatment of carbuncle by injection of carboli acid. Med Record vom 15. Oktober 1892. - 11. Han s Vörner, Ueber die Haltbarmachung von Liquuor aluminium aceticum durch Zusatz von Acid. boric. Münchener medizinische Wochenschrift 1904, No. 24. Konrad Ludwig, Ueber den Gebrauch der 1905 , No. 14. - 12. Hans Vörner, Ueber die Verwendbarkeit der konzentrierten Karbolsăurelosung zur Behandlung des Scrofuloderma und der Furunkulose. Münchener medizinische Wochenschrift 1905, No. 41. 